\title{
Automated engine calibration of hybrid electric vehicles
}

\author{
Nikolce Murgovski, Markus Grahn, Lars Johannesson and Tomas McKelvey
}

\begin{abstract}
We present a method for automated engine calibration, by optimizing engine management settings and power-split control of a hybrid electric vehicle. The problem, which concerns minimization of fuel consumption under a $\mathrm{NO}_{\mathrm{X}}$ constraint, is formulated as an optimal control problem. By applying Pontryagin's maximum principle, this study shows that the problem is separable in space. In the case where the limits of battery state of charge are not activated, we show that the optimization problem is also separable in time. The optimal solution is obtained by iteratively solving the power-split control problem using dynamic programming or the Equivalent Consumption Minimization Strategy. In addition, we present a computationally efficient suboptimal solution, which aims at reducing the number of power-split optimizations required. An example is provided concerning optimization of engine management settings and power-split control of a parallel hybrid electric vehicle.
\end{abstract}

Index Terms-Hybrid electric vehicle, engine management system, engine calibration, optimal control

\section{INTRODUCTION}

Electrification of vehicles is a promising technology that has the potential to improve energy efficiency of vehicles and thereby reduce carbon dioxide emissions. An example of electrified vehicles are hybrid electric vehicles (HEVs). HEVs possess most of the features of conventional vehicles, but besides the internal combustion engine (ICE), they also include an energy buffer, typically a battery, and one or more electric machines (EMs). This gives them an additional degree of freedom in choosing engine operating points, which allows more efficient operation [1].

The energy efficiency of HEVs depends on several factors, from which the most studied are optimal power-split control (see e.g. [1], [2] and references therein), and optimal dimensioning of powertrain components (see e.g. [3], [4], [5], and references therein). The power-split control is a dynamic optimization problem that governs arbitration of demanded power between the ICE and EMs. The problem can be approached in different ways, but besides heuristic and rule-based approaches [6], [7], [8], [9], [10], the dominating approaches contain an optimal control formulation, based on, e.g., dynamic programming (DP), or Pontryagin's maximum principle [1], [7], [11], [12], [13]. The problem formulations often consider idealized conditions, assuming an exact knowledge of the driving mission. The idealized solutions could then

N. Murgovski, M. Grahn, L. Johannesson and T. McKelvey are with the Department of Signals and Systems, Chalmers University of Technology, Gothenburg, Sweden. M. Grahn is also with Volvo Car Corporation. L. Johannesson is also with Viktoria Swedish ICT. Email: nikolce.murgovski@chalmers.se, markus.grahn@volvocars.com, larsjo@chalmers.se, tomas.mckelvey@ chalmers.se

This work has been partially funded by the Swedish Energy Agency. be used as a benchmark for HEV powertrain design, or as a starting point for development of power-split strategies for real-time control. The Equivalent Consumption Minimization Strategy (ECMS) is a well-known real-time control strategy that can be regarded as an approximation of an optimal control formulation [2].

Besides energy efficiency, additional performance criteria in vehicles are the levels of harmful emissions, such as nitrogen oxides $\left(\mathrm{NO}_{\mathrm{X}}\right)$, soot, carbon monoxide, and hydrocarbons, which are combustion byproducts formed in ICEs. An effective solution that reduces toxic emissions in gasoline engines is a three-way-catalyst (TWC). The TWC, however, cannot be used for a Diesel ICE. Instead, Diesel vehicles are equipped with oxidation catalyst and particulate filter, which effectively remove soot, hydrocarbons and carbon monoxide [14]. Unless additionally equipped with a lean $\mathrm{NO}_{\mathrm{X}}$ trap or a selective catalytic reduction, Diesel vehicles cannot reduce the engineout $\mathrm{NO}_{\mathrm{X}}$ emissions. Instead, the approach is to operate the engine such that $\mathrm{NO}_{\mathrm{X}}$ emissions are low already when leaving the engine. During combustion, there is a well-known tradeoff between fuel consumption and $\mathrm{NO}_{\mathrm{X}}$ emissions; control measures that decrease $\mathrm{NO}_{\mathrm{X}}$ emissions typically increase fuel consumption and vice versa [15].

Methods that minimize fuel consumption in an HEV while limiting, or penalizing $\mathrm{NO}_{\mathrm{X}}$ emissions, have been developed and described in literature [7], [16], [17]. However, these studies do not consider the dependence on optimal performance of engine management system settings, concerning boost pressure, exhaust gas recirculation rate, fuel rail pressure, multiple injections with controllable timings and durations, etc. Instead, when optimizing power-split control of HEVs, the ICE's management system is typically set (calibrated) for a conventional vehicle.

A common approach when calibrating engine management settings is to first approximate a given driving cycle to steadystate engine operating points. Then, the ICE calibration at these points is either based on solving the Lagrangian primal [18], or dual problem [19], [20]. In the latter problem, the objective function is essentially a sum of fuel consumption and weighted emissions, using a constant weighting factor (the Lagrange multiplier). Early work with the latter approach can be found in applications of conventional vehicles, for both gasoline ICE [18], [19] and Diesel ICE [20]. Most recent work with this approach has been performed in [21], [22], within the scope of conventional vehicles.

The ICE operation in conventional vehicles and HEVs is different, and the optimal ICE settings in a conventional vehicle are most likely not optimal for the same engine in 
an HEV. (For example, in an HEV, ICE operation at low speed and torque is generally avoided [1].) Moreover, an HEV may include a downsized engine that has been calibrated for a smaller size conventional vehicle, or an engine that is specifically designed for an HEV. Therefore, the ICE calibration in an HEV should be performed with respect to the optimal engine operating points that are obtained by the energy management (power-split) controller. In turn, the optimal power-split control depends on how the ICE is calibrated. These two tasks are strongly coupled and the optimization should reflect on that.

Surprisingly, there are only few published papers on this topic. The published strategies rely on the possibility of splitting the optimization problem in two parts, one for the calibration and one for the power-split control. The ICE calibration is performed similarly as mentioned above for conventional vehicles, while the power-split control is based either on suboptimal methods [23], or on DP [24], [25].

In this study, we revisit the problem of combined ICE calibration and HEV power-split control, and we formulate the problem as a constrained, dynamic optimal control problem, where states are $\mathrm{NO}_{\mathrm{X}}$ mass and battery state of charge (SOC). This allows the problem to be studied using optimal control theory [26], where problem separability in space (optimization variables) is derived directly from Pontryagin's maximum principle [27]. We show that for a special case, where battery SOC limits are not activated, the optimization problem is also separable in time and can be solved efficiently. The optimal solution is obtained by iteratively solving the powersplit control problem using dynamic programming [28] and the ECMS [2]. We present also a computationally efficient suboptimal solution, which aims at reducing the number of power-split optimizations required. An example is provided concerning optimization of engine management settings and power-split control of a parallel hybrid electric vehicle. The computationally efficient solution for the studied example managed to achieve the global optimum in less than 4 minutes.

The paper is outlined as follows. Section II provides background on engine calibration in conventional vehicles, state of the art in engine calibration and delimitations of this study. The problem formulation and modeling details are described in Section III. The optimization method is presented in Section IV. Computationally efficient optimization methods are provided in Section V. An example of ICE calibration and power-split control of an HEV is given in Section VI. The paper is ended with a discussion and a conclusion in Section VII and Section VIII.

\section{ENGINE CALIBRATION PROCEDURES FOR CONVENTIONAL VEHICLES}

Common controllable systems that affect fuel consumption and emissions of a modern passenger car are the gas exchange system, the fuel injection system, and the after-treatment system. These systems have many degrees of freedom, and many settings that can be calibrated. A sophisticated global optimization procedure that accounts for all systems' settings is, in practice, not (yet) possible [29], [30]. Instead, some systems' settings are calibrated manually at an early design stage, and possibly in conjunction with the hardware design, such as design of pistons, cylinder head, and physical location of injectors. The early stage calibration is based on limited input data, typically the operating range of engine speed and load. Examples of settings calibrated at the early stage are the number of injection pulses for combustion events, individual dwell time between different injection pulses, fuel rail pressure, and relative injection amounts in different injection pulses.

Next, the remaining systems' settings are calibrated with respect to a complete driving cycle. Three settings, $z=$ $\left[\begin{array}{lll}z_{1} & z_{2} & z_{3}\end{array}\right]^{T}$, are typically considered, which have a large impact on fuel consumption and emissions. These settings are the injection timing, $z_{1}$, duty cycle to the exhaust gas recirculation valve, $z_{2}$, and duty cycle to the variable geometry turbine, $z_{3}$.

\section{A. Calibration under steady-state operation}

The calibration procedure of $z$ involves two steps at which optimal set points for $z$ are obtained. First, an engine model is generated in a form of five-dimensional static maps for fuel consumption and emissions. The process involves operating the engine in a test cell, for discrete grid values of engine speed, torque, and $z$, within the entire operating range of the engine. Fuel consumption and emissions are measured under stationary conditions.

Second, the optimal set points for $z$ are obtained by minimizing fuel consumption, while fulfilling constraints on accumulated emissions. The optimization is performed over a set of representative engine speed/torque operating points, which are obtained by simulating the vehicle model on a certain driving cycle. Note that, although these speed/torque points may not excite the entire operating range of the engine, they can still be used to optimize the complete working range of the engine. This is a direct result of the optimal calibration procedure, which considers a scalar coefficient weighting the flow of fuel and emissions for the entire operating range of the engine [18], [19], [20]. Obtaining the optimal weighting coefficient is an iterative procedure, where simulation of the vehicle model and optimization of the set points for $z$ is repeated several times. The method will be detailed later, in Section IV-B, for the case of a hybrid electric vehicle. Note that, since the procedure is iterative, the representative operating points are obtained by simulating a vehicle model, rather than operating the real vehicle.

The resulting engine calibration is optimal only for the set of speed/torque points that are chosen as representative. How these points are chosen depends mainly on the standard procedure for determining the legislative limits for emissions. In Europe, the New European Driving Cycle (NEDC) is designed to assess emission levels of passenger vehicles [31].

The set of representative points depends also on the powertrain and engine model. The powertrain model translates the speed/torque points of the driving cycle from the wheels to the engine. It is relevant that these models approximate reasonably well the net fuel consumption and emissions of the real vehicle. The modeling accuracy becomes more important as the new driving cycle, the World-Harmonized Light-Duty 


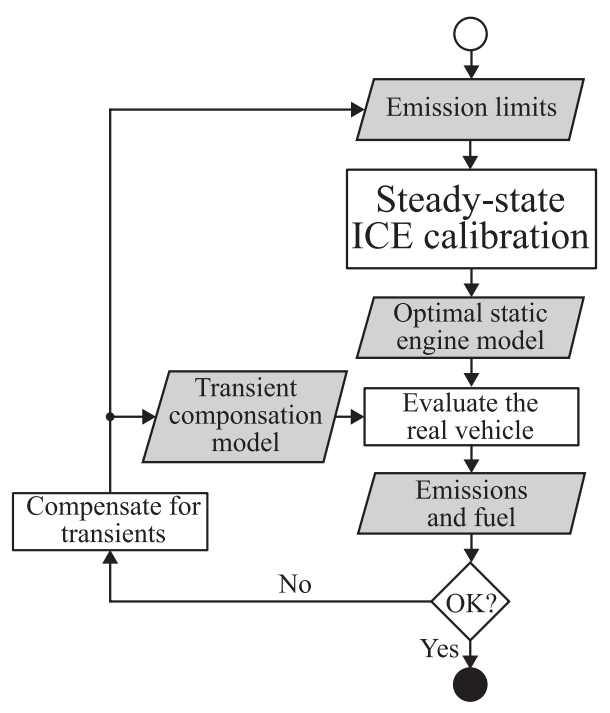

Fig. 1. Optimization framework for steady-state engine calibration, including compensations for transients. The steady-state calibration is repeated several times, after adjusting (typically manually) the emission limits and the transient compensation model.

Vehicles Test Cycle, is being developed. This cycle is likely to include a larger portion of engine transient operation, compared to the NEDC [31].

\section{B. Compensations for transients}

When calibrating the engine based on a static engine model, the transition between two successive operating points is assumed immediate. However, dynamics in the engine necessitate a transient engine operation, which leads to higher fuel consumption and emissions than fuel consumption and emissions from the static engine model. To account for the differences, compensations are used that limit emissions during transients [32], [33], [34], [35]. The compensations involve manual adjustments of the optimal engine set points, typically the oxygen fraction in the intake manifold and the injection timing. Additionally, when calibrating the static engine model, the emissions' limits are lowered, such that the real vehicle does not exceed the legislative limits for emissions.

After the transient compensations are performed, the static engine model is recalibrated. The whole process is iterated several times with different limits on accumulated emissions, until the real vehicle achieves satisfactory fuel consumption and emission levels. The engine calibration process, including transient compensation, is illustrated in Fig. 1.

\section{State of the art in engine calibration and delimitations of this study}

The research in engine management system optimization is divided into optimization of steady-state engine operation and transient engine operation. The state of the art optimization of steady-state engine operation is as described in Section II-A.

The state of the art research on optimization of transient engine operation has been mainly focused on reducing emission spikes for a subset of engine transient scenarios, rather than optimizing fuel consumption on a complete driving cycle

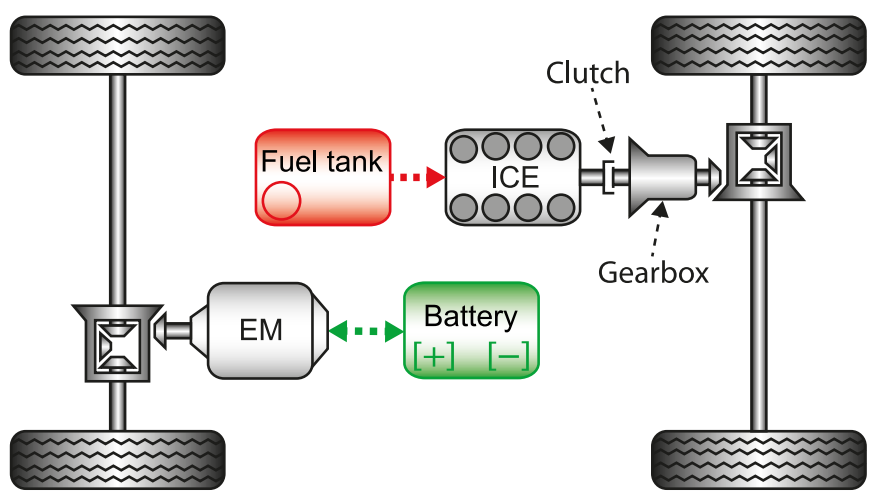

Fig. 2. Hybrid electric vehicle with a parallel powertrain configuration. The internal combustion engine propels the front wheels, while the electric machine is mounted on the rear axle.

[36], [37], [38], [39], [40], [41]. Several studies have included optimization over a complete driving cycle, considering both steady-state and transient engine operation [32], [33], [42], [43]. The approach in [42], [43] proposes neural network for modeling transient engine behavior, while the approach in [32], [33] is complementing transient compensations, rather than replacing them with an accurate dynamic engine model. Optimal engine calibration based on a complete dynamic engine model is yet to be developed.

The study presented in this paper focuses solely on engine calibration based on a static engine model. The transient compensation procedure, which is well established in literature, is not further discussed in the rest of this paper.

\section{POWERTRAIN MODEL AND PROBLEM FORMULATION}

Depending on the ICE and EM arrangement, HEV powertrains are commonly divided into series, parallel and seriesparallel configurations [1]. The powertrain studied here is a through-the-road parallel powertrain, in which both the ICE and EM are mechanically connected to the wheels. The powertrain is constructed by augmenting a front-wheel driven conventional vehicle with a battery and an EM mounted on the rear axle, as illustrated in Fig. 2. The EM can operate in both motoring and generating mode. This allows recuperation of braking energy, which in the case of a conventional vehicle is lost as heat.

\section{A. Powertrain model}

The vehicle is required to fulfill a certain driving mission fully described by road altitude, desired vehicle velocity and acceleration at each point in time. In the view of the vehicle powertrain, this can be translated to angular velocity $\omega$ and torque $T$ demanded at the wheels, for each time instant of the driving mission. When delivering the inputs $\omega$ and $T$, we adopt a commonly used backward-simulation, quasi-static powertrain model [1], which does not necessitate a driver model. In this model, the vehicle follows exactly the reference 

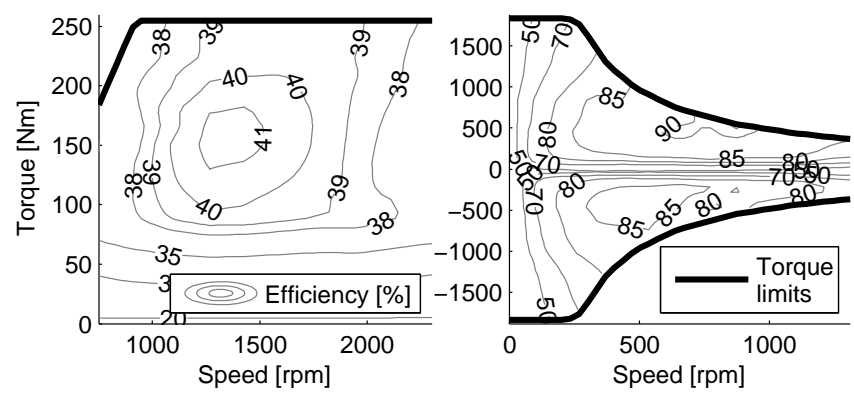

Fig. 3. Static maps of an ICE, left, and an EM, right. The ICE map depicts efficiency for fixed values for injection timing, duty cycle to the exhaust gas recirculation valve and duty cycle to the variable geometry turbine. The EM efficiency map includes also the losses from the inverter and rear differential gear.

trajectories, $\omega$ and $T$, yielding the following equations

$$
\begin{aligned}
& T=T_{E} r(g) \eta(g)+T_{M}, \\
& P_{B}=T_{M} \omega+P_{M d}\left(T_{M}, \omega\right), \\
& \omega_{E}=r(g) \omega .
\end{aligned}
$$

The notation above reads as follows: $T_{E}$ and $T_{M}$ are torques of the ICE and EM, $\omega_{E}$ is rotational speed of the ICE, $r$ and $\eta$ are gear ratio and efficiency of gear $g$, and $P_{B}$ is power at the battery terminals. The efficiencies of all gears, including the two differential gears, are modeled as constant. The efficiency and gear ratio of the front differential gear are denoted with $\eta$ and $r$, while the losses of the rear differential gear and power inverter are reflected within the EM losses $P_{M d}$. The dominating inertia of the vehicle is the inertia of the vehicle itself (chassis, driving axels, wheels, etc.), and we have therefore neglected the rotational inertia of the ICE and EM. Auxiliary load is not considered.

The battery is modeled as a series connection of a voltage source $u(s)$ and a resistance $R(s)$, which, in a general case, are both a function of state of charge (SOC). Then, the SOC derivative is given as

$$
\dot{s}=-\frac{i}{Q_{B}}=f_{s}\left(P_{B}, s\right),
$$

with

$$
f_{s}\left(P_{B}, s\right)=-\frac{u(s)-\sqrt{u^{2}(s)-4 R(s) P_{B}}}{2 Q_{B} R(s)} .
$$

In the equations above, $s$ and $Q_{B}$ denote battery SOC and capacity, respectively. Equation (5) is obtained by deriving the battery current $i$ from

$$
P_{B}=u(s) i-R(s) i^{2} .
$$

The ICE is modeled by two static functions, $f_{f}\left(\omega_{E}, T_{E}, z\right)$ and $f_{N O_{X}}\left(\omega_{E}, T_{E}, z\right)$, describing fuel consumption and $\mathrm{NO}_{\mathrm{X}}$ emissions in $\mathrm{g} / \mathrm{s}$, respectively. The inputs $z=\left[z_{1} z_{2} z_{3}\right]^{T}$, are control variables denoting injection timing, $z_{1}$, duty cycle to the exhaust gas recirculation valve, $z_{2}$, and duty cycle to the variable geometry turbine, $z_{3}$. (See, e.g., [15] for background on ICEs.) An example of the ICE's efficiency map for fixed values of $z$ is given in Fig. 3. The maps' generation is further discussed in Section VII-A.
Similarly, the EM's dissipative power $P_{M d}$, including the inverter and rear differential losses, is obtained from speed/torque measurements under stationary conditions. An example of a static EM model is given in Fig. 3.

To keep the problem simple it is assumed that no additional losses are associated with arbitration of the engine on/off state, gear selection, and clutch engagement. Therefore, when the engine does not deliver torque it is turned off and declutched from the wheels. This does not affect the generality of the solution proposed in Section IV, as the method allows inclusion of dynamic engine on/off, transmission and clutch model. This is further discussed in Section VII-B.

\section{B. Problem formulation}

The optimization objective is formulated to minimize fuel consumption, by limiting $\mathrm{NO}_{\mathrm{X}}$ emissions under a certain level

$$
\int_{0}^{t_{f}} f_{N O_{X}}\left(\omega_{E}, T_{E}, z\right) d t \leq m_{N O_{X} \max }
$$

throughout the entire driving mission with duration $t_{f}$. Then, the optimization problem can be formulated as follows:

$$
\begin{aligned}
& \min _{g, T_{E}, z} \int_{0}^{t_{f}} f_{f}\left(\omega_{E}, T_{E}, z\right) d t, \\
& \text { subject to: } \\
& T=T_{E} r(g) \eta(g)+T_{M}, \\
& P_{B}=T_{M} \omega+P_{M d}\left(T_{M}, \omega\right), \\
& \omega_{E}=r(g) \omega . \\
& \dot{m}_{N O_{X}}=f_{N O_{X}}\left(\omega_{E}, T_{E}, z\right), \\
& m_{N O x} \in\left[0, m_{N O_{X} \max }\right], \\
& z \in \mathcal{Z}\left(\omega_{E}, T_{E}\right), \\
& \dot{s}=f_{s}\left(P_{B}, s\right), \\
& s(0)=s\left(t_{f}\right)=s_{0}, \\
& s \in\left[s_{\min }, s_{\max }\right], \\
& T_{M} \in\left[T_{M \min }(\omega), T_{M \max }(\omega)\right], \\
& T_{E} \in\left[0, T_{\text {Emax }}\left(\omega_{E}\right)\right], \\
& \omega_{E} \in\left[\omega_{\text {Eidle }}, \omega_{\text {Emax }}\right], \\
& P_{B} \in\left[P_{B \min }, P_{B \max }\right], \\
& g \in\left\{0,1, \ldots, g_{\text {max }}\right\} .
\end{aligned}
$$

The $\mathrm{NO}_{\mathrm{X}}$ constraint (7) has been replaced by equivalent constraints (8e) and (8f). There are five time dependent variables, $g, T_{E}$ and $z=\left[z_{1} z_{2} z_{3}\right]^{T}$, and two states, $m_{N O_{X}}$ and $s$. The vectors $T_{M}, P_{B}$ and $\omega_{E}$ are removed from the control signals in (8). These vectors can be directly expressed by $T_{E}, g$, and the reference signals $T$ and $\omega$, by simply backsubstituting the equalities (8b)-(8d). The constraint (8i) is introduced to conserve battery energy at the beginning and end of the driving cycle. The set $\mathcal{Z}\left(\omega_{E}, T_{E}\right)$ in $(8 \mathrm{~g})$ is a feasible set for $z$, expressed as a function of engine speed and torque. The remaining constraints are boxing constraints on the optimization variables, where the EM and ICE torque limits, (8k) and (81), are functions of speed. All variables have real values, except the gear number $g$, which accepts non-negative integer values. The constraints are imposed for $\forall t \in\left[0, t_{f}\right]$. 


\section{OPTIMIZATION METHOD}

A straightforward way to solve (8) is by directly using dynamic programming (DP). However, a serious limitation of DP is that the computational time increases exponentially with the number of state variables [28]. Recall that the optimization problem (8) has two real-valued states. Solving this problem with a standard DP implementation will require high computational demands. In order to shorten computational time, we propose here an alternative solution based on Pontryagin's maximum principle, which will eliminate at least one state from the problem.

\section{A. Problem separability in space}

The Hamiltonian of the optimization problem (also known as Pontryagin's $\mathcal{H}$ function), reads as follows

$$
\begin{aligned}
\mathcal{H}(\cdot) & =f_{f}\left(\omega_{E}, T_{E}, z\right) \\
& +\lambda_{s} f_{s}\left(P_{B}, s\right)+\lambda_{N O_{X}} f_{N O_{X}}\left(\omega_{E}, T_{E}, z\right) .
\end{aligned}
$$

Here, $\lambda_{s}$ and $\lambda_{N O_{X}}$ are costates of the system, for which it holds

$$
\dot{\lambda}_{s}^{*}=-\left(\frac{\partial \mathcal{H}(\cdot)}{\partial s}\right)^{*}, \quad \dot{\lambda}_{N O_{X}}^{*}=-\left(\frac{\partial \mathcal{H}(\cdot)}{\partial m_{N O_{X}}}\right)^{*}=0,
$$

at any optimal solution (marked by ${ }^{*}$ ). This is a necessary condition at any local/global optimum [26], where the above mentioned derivatives are defined. Hence, the solution of (8) can also be obtained by minimizing the Hamiltonian, i.e.

$$
\begin{aligned}
& \min _{g, T_{E}, z} \mathcal{H}(\cdot), \\
& \text { s.t.: }(8 \mathrm{~b})-(8 \mathrm{o}), \\
& \dot{\lambda}_{s}=-\frac{\partial \mathcal{H}(\cdot)}{\partial s}, \\
& \dot{\lambda}_{N O_{X}}=0 .
\end{aligned}
$$

The necessary condition (10) reveals that along sections of the driving mission, where the derivative $\partial \mathcal{H}(\cdot) / \partial m_{N O_{X}}$ is defined, $\lambda_{N O_{X}}^{*}$ is, in fact, a constant value, because $\mathcal{H}(\cdot)$ does not explicitly depend on $m_{N O_{X}}$. It is clear that the derivative is not defined when the constraint (8f) is active. Since $m_{N O_{X}}$ is monotonically increasing (in time), the constraint (8f) might be active only at two time segments, one starting at the beginning of the driving mission, and one ending at the end of the driving mission. Hence, if there is at least one time instant in the remaining time interval (where $m_{N O_{X}}$ does not lie on the bounds), it is possible to obtain a single constant value for $\lambda_{N O_{X}}^{*}$. (The trivial case where the HEV is operated as a pure electric vehicle along the entire mission is not of interest in this study and has been neglected.) Then, obtaining the optimal value for $\lambda_{N O_{X}}$ is a straightforward procedure, which will be detailed later, in Section IV-D.

Now consider the case where the optimal costate $\lambda_{N O_{X}}^{*}$ is given. (Naturally, we also consider a feasible optimization problem.) The consequence is that the $\mathrm{NO}_{\mathrm{X}}$ limit (8f) can be removed from the problem, as the optimal costate must satisfy this constraint. Without the $\mathrm{NO}_{\mathrm{X}}$ limits, the $\mathrm{NO}_{\mathrm{X}}$ state constraint $(8 \mathrm{e})$ is also not needed and can be removed from the problem. Then, the variables $z$ in (11) are present only in the objective function (9) and in the constraint $(8 \mathrm{~g})$. This allows the problem (11) to be formulated as a bilevel program

$$
\begin{aligned}
& \min _{g, T_{E}} f_{f}\left(\omega_{E}, T_{E}, z^{*}\right)+\lambda_{s} f_{s}\left(P_{B}, s\right) \\
& +\lambda_{N O_{X}}^{*} f_{N O_{X}}\left(\omega_{E}, T_{E}, z^{*}\right), \\
& \text { s.t.: (8b)-(8d), (8h)-(8o), (11b), } \\
& z^{*}=\underset{z}{\operatorname{argmin}} f_{f}\left(\omega_{E}^{*}, T_{E}^{*}, z\right) \\
& +\lambda_{N O_{X}}^{*} f_{N O_{X}}\left(\omega_{E}^{*}, T_{E}^{*}, z\right), \\
& \text { s.t.: } z \in \mathcal{Z}\left(\omega_{E}^{*}, T_{E}^{*}\right)
\end{aligned}
$$

that can be separated into two tasks, by obtaining $z^{*}$ for any feasible combination of $\omega_{E}$ and $T_{E}$. The upper-level task optimizes the HEV power-split control, while the lower-level task optimizes the ICE calibration.

\section{B. Optimal ICE calibration}

The lower-level task in (12), i.e. the engine calibration problem, can be solved independently of the upper-level task, by optimizing $z$ for any feasible combination of values of the optimization variables in the upper-level task. Furthermore, since the engine calibration does not explicitly depend on gear number, and the gear number is directly related to engine speed via (3), $z$ can be optimized for feasible combinations of $\omega_{E}$ and $T_{E}$. Then, the engine calibration problem can be approached by gridding the feasible sets for $z, \omega_{E}$ and $T_{E}$, and solving

$$
\begin{aligned}
& f_{z}^{*}\left(\omega_{E}, T_{E}\right)=\underset{z}{\operatorname{argmin}} f_{f}\left(\omega_{E}, T_{E}, z\right) \\
& \quad+\lambda_{N O_{X}}^{*} f_{N O_{X}}\left(\omega_{E}, T_{E}, z\right), \\
& \text { s.t.: } z \in \mathcal{Z}_{d}\left(\omega_{E}, T_{E}\right), \\
& \omega_{E} \in \mathcal{W}_{E} \subseteq\left[\omega_{E i d l e}, \omega_{E \max }\right] \subseteq \mathbb{R}^{n}, \\
& T_{E} \in \mathcal{T}_{E}\left(\omega_{E}\right) \subseteq \mathbb{R}^{m},
\end{aligned}
$$

where the sets $\mathcal{Z}_{d}, \mathcal{W}_{E}$ and $\mathcal{T}_{E}$ are discrete. Here, $f_{z}^{*}\left(\omega_{E}, T_{E}\right) \in \mathbb{R}^{n \times m \times 3}$ is a three-dimensional map, illustrated in Fig. 4, holding the optimal set points of $z$ for all speed/torque combinations. As a consequence, the fivedimensional fuel and $\mathrm{NO}_{\mathrm{X}}$ maps can be replaced with the two-dimensional maps

$$
\begin{aligned}
& \tilde{f}_{f}\left(\omega_{E}, T_{E}\right)=f_{f}\left(\omega_{E}, T_{E}, f_{z}^{*}\left(\omega_{E}, T_{E}\right)\right), \\
& \tilde{f}_{N O_{X}}\left(\omega_{E}, T_{E}\right)=f_{N O_{X}}\left(\omega_{E}, T_{E}, f_{z}^{*}\left(\omega_{E}, T_{E}\right)\right),
\end{aligned}
$$

which are calibrated with the optimal set points for $z$. The map (14) is used in the power-split optimization problem, to be explained in Section IV-C, for obtaining the optimal engine speed and torque trajectories $\omega_{E}^{*}$ and $T_{E}^{*}$. The optimal engine settings are here obtained as a function of engine speed and torque, but, if needed, they can also be obtained as explicit functions of time, $z^{*}=f_{z}^{*}\left(\omega_{E}^{*}, T_{E}^{*}\right)$, as originally stated in (8). For speed/torque points that are not in the discrete sets $\mathcal{W}_{E}$ and $\mathcal{T}_{E}, z_{\tilde{f}}^{*}$ can be obtained by interpolation in $f_{z}^{*}$.

The maps $\tilde{f}_{f}\left(\omega_{E}, T_{E}\right)$ and $\tilde{f}_{N O_{X}}\left(\omega_{E}, T_{E}\right)$ are calibrated optimally, only when the optimal costate $\lambda_{N O_{X}}^{*}$ is given. For any different value $\lambda_{N O_{X}}$, the problem (13) will generate suboptimal maps. The optimization framework for obtaining $\lambda_{N O_{X}}^{*}$ is described in Section IV-D. 


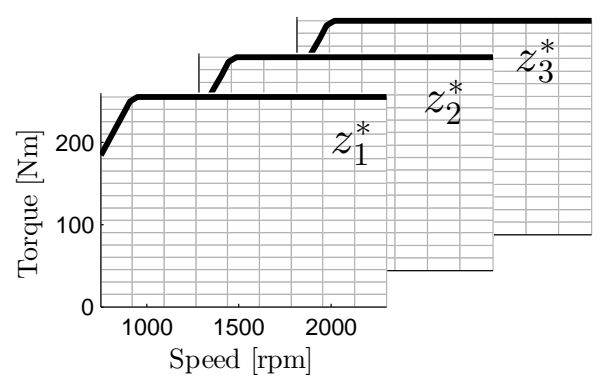

Fig. 4. Illustration of the map $f_{z}^{*}\left(\omega_{E}, T_{E}\right)$ holding the optimal engine calibration points for grid values of engine speed and torque.

\section{Optimal power-split control}

After the engine is calibrated, the power-split control problem is solved by minimizing the Hamiltonian (11), or the primal problem (8), without considering constraints associated to $z$. A problem formulation based on (8) can be written as

$$
\begin{gathered}
\min _{g, T_{E}} \int_{0}^{t_{f}} \tilde{f}_{f}\left(\omega_{E}, T_{E}\right) d t, \\
\text { s.t.: }(8 \mathrm{~b})-(8 \mathrm{~d}),(8 \mathrm{~h})-(8 \mathrm{o}) .
\end{gathered}
$$

Bellman's principle of optimality can be applied for solving the problem via backwards recursion, in a standard DP formulation [28]. Denoting with $J_{D P}^{*}\left(s\left(t_{k}\right), t_{k}\right)$ the cost matrix holding the optimal cost-to-go from state $s\left(t_{k}\right)$ at time $t_{k}$ to the desired final state at time $t_{f}$, the optimization problem, at a time instance $t_{k}$, can be formulated as

$$
\begin{gathered}
J_{D P}^{*}\left(s\left(t_{k}\right), t_{k}\right) \\
=\min _{g\left(t_{k}\right), T_{E}\left(t_{k}\right)}\left\{\tilde{f}_{f}\left(\omega_{E}\left(t_{k}\right), T_{E}\left(t_{k}\right)\right) \Delta t\right. \\
\left.\quad+J_{D P}^{*}\left(s\left(t_{k+1}\right), t_{k+1}\right)\right\}, \\
\text { s.t.: }(8 \mathrm{~b})-(8 \mathrm{~d}),(8 \mathrm{k}),(8 \mathrm{~m})-(8 \mathrm{o}) \text { at } t_{k}, \\
s\left(t_{k+1}\right)-s\left(t_{k}\right)=f_{s}\left(P_{B}\left(t_{k}\right), s\left(t_{k}\right)\right) \Delta t \\
s\left(t_{k}\right) \in \mathcal{S} \subseteq\left[s_{\min }, s_{\max }\right] \\
T_{E}\left(t_{k}\right) \in \mathcal{T}_{E}\left(\omega_{E}\left(t_{k}\right)\right) \\
t_{k} \in \mathcal{T} \subseteq\left[0, t_{f}\right] .
\end{gathered}
$$

Discrete values are used for time, battery SOC and ICE torque, and the SOC derivative is replaced with a difference. The grid resolution of the discrete sets, $\mathcal{T}, \mathcal{S}$ and $\mathcal{T}_{E}$, is a tradeoff between computational time and accuracy. The sampling interval is denoted by $\Delta t$.

The cost at the final time is a penalty for violating the battery charge sustaining constraint. A typical choice, used later in Section VI, is a linear penalty function

$$
J_{D P}^{*}\left(s\left(t_{f}\right), t_{f}\right)=L \cdot\left|s\left(t_{f}\right)-s_{0}\right|
$$

where $L$ is a large positive number.

\section{Optimization framework}

Finally, we present an algorithm for obtaining the optimal solution of (8). The procedure is based on iteratively calibrating the ICE and solving the power-split control problem for

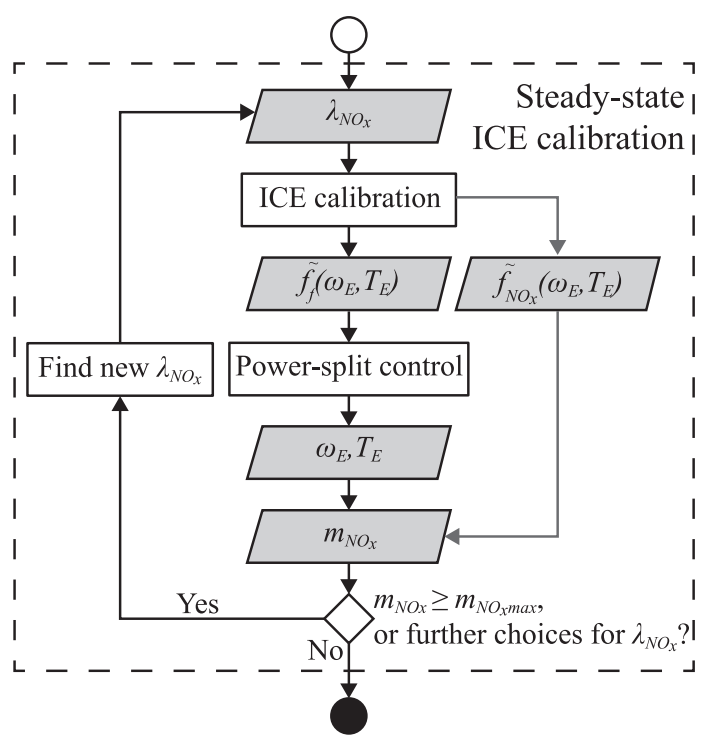

Fig. 5. Optimization framework for ICE calibration in a hybrid electric vehicle. The ICE calibration is an iterative procedure, where iterations are needed for obtaining the optimal costate that weights the tradeoff between fuel consumption and emissions.

several values of $\lambda_{N O_{X}}$, until the optimal $\lambda_{N O_{X}}^{*}$ is attained. The set of steps to be performed is illustrated in Fig. 5 and line up as follows: for an initial guess of $\lambda_{N O_{X}}$ the ICE is calibrated by solving (13) and the two-dimensional maps $\tilde{f}_{N O_{X}}\left(\omega_{E}, T_{E}\right)$ and $\tilde{f}_{f}\left(\omega_{E}, T_{E}\right)$ are obtained. The latter map is used in the DP problem (17), to obtain the optimal engine speed and torque trajectories. Then, using the optimal trajectories the $\mathrm{NO}_{\mathrm{X}}$ emission and fuel consumption are computed. This procedure is repeated for several values of $\lambda_{N O_{X}}$ and the solution is kept that gives the lowest fuel consumption that does not violate the $\mathrm{NO}_{\mathrm{X}}$ limit (8f).

There are several ways to obtain $\lambda_{N O_{X}}^{*}$. A straightforward way is to grid the set of costate values and evaluate the algorithm for each discrete value. The costate is nonnegative, while an approximate upper bound can be found by minimizing $\mathrm{NO}_{\mathrm{X}}$ emissions for any feasible $z$, or by engineering intuition. A more efficient way to obtain the optimal costate is to use the contradictive nature of the two objectives; that is, higher penalty for $\mathrm{NO}_{\mathrm{X}}$ emissions leads to increased fuel consumption. Therefore, a monotonic dependence of fuel consumption on $\lambda_{N O_{X}}$ can be expected; this has also been observed in the example in Section VI. The monotonic behavior can be exploited to efficiently obtain $\lambda_{N O_{X}}^{*}$ by a root finding algorithm, e.g. bisection.

\section{COMPUTATIONALly EFFICIENT SOLUTIONS}

A heavy computational burden in the engine calibration framework is the iterative solutions of the DP problem (17). In this section we propose solutions that require fewer powersplit optimizations, or replace the power-split control with a time efficient alternative.

\section{A. Reversed bilevel program}

Consider the bilevel program (12), where the lower-level task is moved to the upper level, and the upper-level task is 


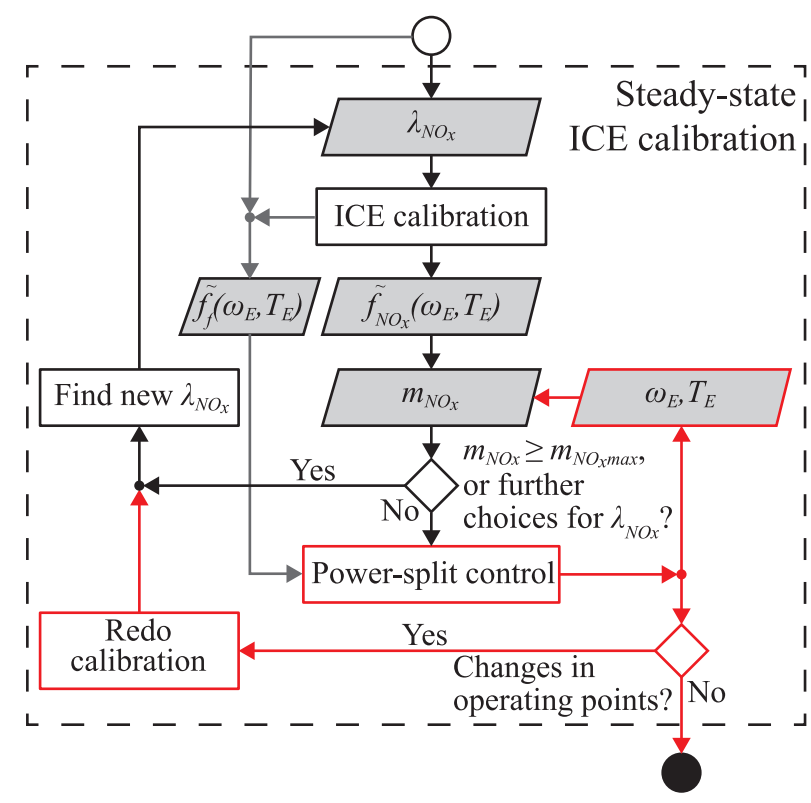

Fig. 6. Optimization framework for computationally efficient ICE calibration in a hybrid electric vehicle. The power-split control is taken outside the inner ICE calibration loop. The procedure requires fewer power-split optimizations, but it may deliver a local optimum.

moved to the lower level. The resulting bilevel program

$$
\begin{aligned}
& \min _{z} f_{f}\left(\omega_{E}^{*}, T_{E}^{*}, z\right)+\lambda_{N O_{X}}^{*} f_{N O_{X}}\left(\omega_{E}^{*}, T_{E}^{*}, z\right), \\
& \text { s.t.: } z \in \mathcal{Z}\left(\omega_{E}^{*}, T_{E}^{*}\right), \\
& \omega_{E}^{*}=r\left(g^{*}\right) \omega, \\
& {\left[g^{*}, T_{E}^{*}\right]=\underset{g, T_{E}}{\operatorname{argmin}} f_{f}\left(\omega_{E}, T_{E}, z^{*}\right)+\lambda_{s} f_{s}\left(P_{B}, s\right)} \\
& \quad \text { s.t.: (8b)-(8d), (8h)-(8o), (11b), }
\end{aligned}
$$

is not easily separable in space, as it would require solving the power-split problem for all feasible combinations of $z$. Instead, this formulation is used as a starting point for a computationally efficient, locally-optimal solution.

A computationally efficient strategy can be devised by assuming that a map $f_{z 0}\left(\omega_{E}, T_{E}\right)$ is given, which is, preferably, in the neighbourhood of $f_{z}^{*}\left(\omega_{E}, T_{E}\right)$. Then, the map $\tilde{f}_{f 0}\left(\omega_{E}, T_{E}\right)$ is obtained from (14) and the power-split control in the lower level task in (19) [or equivalently (16)] delivers the representative points $\omega_{E 0}^{*}, T_{E 0}^{*}$. The representative points are used for recalibrating the ICE, giving new maps $\tilde{f}_{f 1}\left(\omega_{E}, T_{E}\right)$, $\tilde{f}_{N O_{X} 1}\left(\omega_{E}, T_{E}\right)$. The difference from the optimization framework in Fig. 5 is that the power-split problem is moved outside the loop for obtaining $\tilde{f}_{f 1}\left(\omega_{E}, T_{E}\right), \tilde{f}_{N O_{X} 1}\left(\omega_{E}, T_{E}\right)$, where iterations over $\lambda_{N O_{X}}$ are performed.

The procedure repeats until convergence, or maximum number of iterations is reached. The advantage of this strategy is that fewer power-split optimizations might be required. The disadvantage is that the solution may not converge to the global optimum, as there is no easy way to choose $f_{z 0}\left(\omega_{E}, T_{E}\right)$ sufficiently close to $f_{z}^{*}\left(\omega_{E}, T_{E}\right)$.

A flowchart of the optimization framework is presented in Fig. 6.

\section{B. Problem separability in time}

The power-split control problem has been widely studied in literature, both for assessment of performance, and for realtime model predictive control of HEVs. In this section a computationally efficient power-split control is briefly discussed. In a general case this solution is suboptimal, although an optimal result could be achieved in special cases.

A well known time efficient power-split control that has been observed to give near optimal results is the ECMS [2], [44], [45]. The basic assumption behind ECMS is that the optimal battery SOC costate $\lambda_{s}^{*}$ does not vary significantly from a certain reference value (typically constant), which could be obtained with connection to the typical daily usage of the vehicle. Operating with the reference costate may cause violation of the battery SOC limits, and therefore $\lambda_{s}$ is allowed to deviate from the reference as the battery SOC gets close to the limits. Typical approach is to control $\lambda_{s}$ with some sort of closed loop controller [46], [47], [48].

A special case of the ECMS is a power-split control of a vehicle with a large battery, where the battery SOC limits are not activated at any time instant along the driving cycle. Then, the obtained solution is indeed optimal, if an initial $\lambda_{s 0}^{*}$ can be found, for which the optimal solution of (11) satisfies the charge sustaining constraint (8i), without activating the SOC limits (8j). As it turns out, the scenario where SOC limits are not activated is a typical case in the energy management of HEVs (see e.g. [1], [2] and references therein). Then, the power-split control problem

$$
\begin{aligned}
& {\left[g^{*} T_{E}^{*}\right]=\underset{g, T_{E}}{\operatorname{argmin}} \tilde{f}_{f}\left(\omega_{E}, T_{E}\right)+\lambda_{s} f_{s}\left(P_{B}, s\right), } \\
& \text { s.t.: (8b)-(8d), (8h), (8k)-(8o), (11b), } \\
& \lambda_{s}(0)=\lambda_{s 0}^{*},
\end{aligned}
$$

becomes separable in time. This means that the optimal control signals $g^{*}\left(t_{k}\right), T_{E}^{*}\left(t_{k}\right)$, at some time instant $t_{k}$, can be obtained by instantaneous optimization of (20), considering only the single time instant $t=t_{k}$. This is a direct consequence from the removal of the SOC limits and the charge sustaining constraint. The optimal solution can be obtained in a forward simulation manner. First, for the given $\lambda_{s 0}^{*}$, the optimal controls $g^{*}(0), T_{E}^{*}(0)$ are obtained by solving (20) at $t=0$. At the same time, both the battery state and costate are integrated, thus obtaining $s^{*}\left(t_{1}\right)$ and $\lambda_{s}^{*}\left(t_{1}\right)$, at the next time instant $t_{1}$. Then, the optimal control signals $g^{*}\left(t_{1}\right), T_{E}^{*}\left(t_{1}\right)$ are obtained by solving (20) at $t=t_{1}$. The procedure repeats until $t=t_{f}$ is reached.

The challenge in this strategy is obtaining $\lambda_{s 0}^{*}$ that satisfies the SOC sustaining constraint (8i). The method is similar to the one obtaining $\lambda_{N O_{X}}^{*}$. The power-split problem (20) is iteratively solved for different values of $\lambda_{s 0}$, where in each iteration the optimal control is obtained for all time instances. The procedure stops when $s\left(t_{f}\right)$ is close to $s_{0}$. Bisection can also be applied for obtaining a new value for $\lambda_{s 0}$.

The optimization framework of the power-split control based on the ECMS is illustrated in Fig. 7. This power-split control can be used in combination with the global optimization framework depicted in Fig. 5, and in the framework for computationally efficient ICE calibration depicted in Fig. 6. 


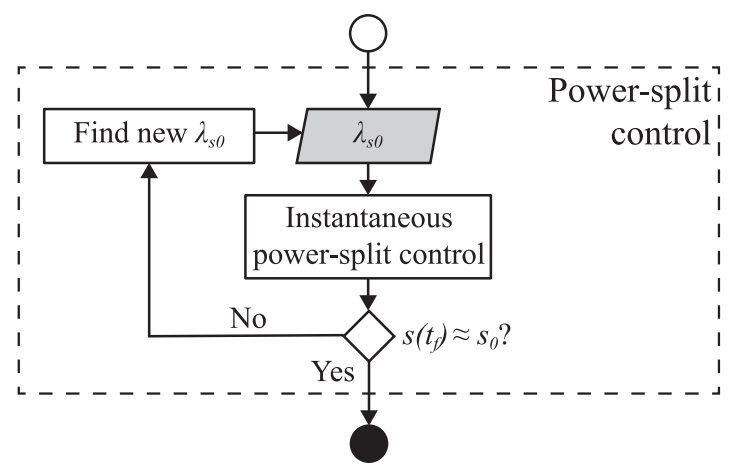

Fig. 7. Optimization framework for computationally efficient power-split control of a hybrid electric vehicle, based on the Equivalent Consumption Minimization Strategy (ECMS). The optimization is iterative, where iterations are needed for obtaining the optimal initial value for the battery SOC costate.

\section{OPTIMIZATION EXAMPLE}

This section provides an optimization example of ICE calibration and power-split control of a through-the-road parallel HEV. We consider a vehicle with $60 \mathrm{~kW}$ Diesel ICE and $\pm 50 \mathrm{~kW}$ EM, as illustrated in Fig. 3. The five-dimensional ICE's fuel and $\mathrm{NO}_{\mathrm{X}}$ flow maps are obtained using steadystate measurements. The Lithium-ion battery is modeled by constant open circuit voltage and resistance. Its available energy is $464 \mathrm{Wh}$, which is half of its total capacity; to mitigate battery wear the battery is operated within $25-75 \%$ SOC. We require the initial and final SOC of the battery to be $50 \%$. Emission levels are assessed using both the New European Driving Cycle (NEDC), and the USA's Federal Test Procedure $^{1}$ (FTP75). The vehicle is required to emit not more than $0.18 \mathrm{~g} / \mathrm{km} \mathrm{NO}_{\mathrm{X}}$ on the NEDC, which corresponds to Euro $\mathrm{V}$ targets on $\mathrm{NO}_{\mathrm{X}}$ emissions. For simplicity, the same level of $0.18 \mathrm{~g} / \mathrm{km}$ is kept for FTP75.

\section{A. Computational performance}

The optimization problem is solved using the framework illustrated in Fig. 5, where the power-split control is optimized by the ECMS, using 300 discrete points for the ICE torque. Without considering discretization error, the ECMS result is optimal, since the optimal battery SOC does not activate SOC limits, and the optimal SOC costate can be found that satisfies the SOC charge sustaining constraint. The satisfaction of the battery SOC constraints can be observed in Fig. 9. Furthermore, since the battery is modeled by a constant open circuit voltage and resistance, it follows from the necessary condition

$$
\dot{\lambda}_{s}^{*}=-\left(\frac{\partial \mathcal{H}(\cdot)}{\partial s}\right)^{*}=0
$$

that the optimal SOC costate is a constant scalar value, since $\mathcal{H}(\cdot)$ does not explicitly depend on $s$. The dependence of SOC costate on $\mathrm{NO}_{\mathrm{X}}$ costate, i.e. the dependence of Diesel consumption on $\mathrm{NO}_{\mathrm{X}}$ emissions, is shown in Fig. 8, for different values of the difference $s\left(t_{f}\right)-s_{0}$.

\footnotetext{
${ }^{1}$ The driving cycles, NEDC and FTP75, are available online at http://www.dieselnet.com/standards/cycles. May 2014.
}
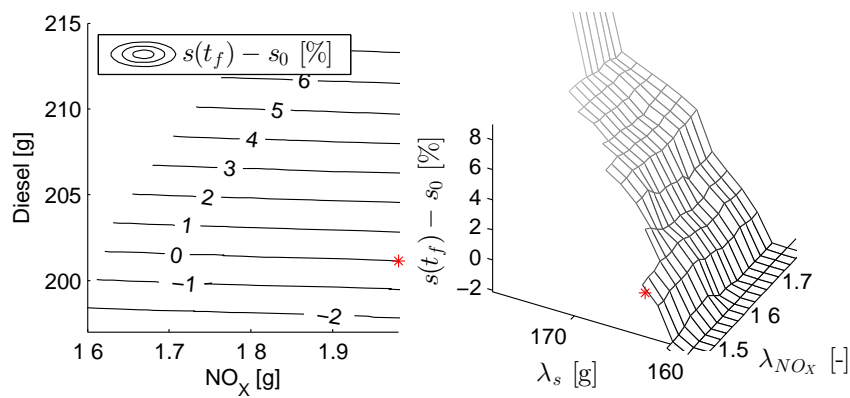

Fig. 8. Diesel consumption vs. $\mathrm{NO}_{\mathrm{X}}$ emissions for various deviations from the desired final SOC. The left plot is a contour plot, while the right plot shows an alternative view, a surface plot, of the influence of $\mathrm{NO}_{\mathrm{X}}$ and SOC costates on deviation from the desired final SOC. The optimal result is indicated by the star.

TABLE I

DIESEL CONSUMPTION AND NO $\mathrm{X}_{\mathrm{X}}$ EMISSIONS FOR THE CONVENTIONAL VEHICLE, THE HEV WITH ICE CALIBRATED FOR THE CONVENTIONAL VEHICLE, AND THE HEV WITH THE OPTIMALLY CALIBRATED ICE.

\begin{tabular}{lcccc}
\hline & \multicolumn{2}{c}{ NEDC } & \multicolumn{2}{c}{ FTP75 } \\
Quantity [g] & Diesel & $\mathrm{NO}_{\mathbf{X}}$ & Diesel & $\mathrm{NO}_{\mathbf{X}}$ \\
Conventional vehicle & 276.67 & 1.97 & 473.17 & 3.20 \\
HEV (conventional ICE) & 202.38 & 1.25 & 301.81 & 2.16 \\
HEV (recalibrated ICE) & 201.13 & 1.97 & 299.92 & 3.19 \\
$\mathrm{NO}_{\mathbf{X}}$ limit & & 1.97 & & 3.20 \\
\hline
\end{tabular}

The number of investigated costate values depends on the the desired accuracy and initial knowledge of the interval in which the optimal costates reside. An engineering estimate is that not more than $40 \times 40$ iterations are needed until the optimal solution is obtained with a relative error of less than $0.01 \%$ in both fuel consumption and SOC sustenance (the measurement and discretization errors, for example, are expected to be larger). The entire problem is solved in less than 100 minutes, on a standard PC (4 GB RAM, $2.67 \mathrm{GHz}$ dual core $\mathrm{CPU})$. The computational time can be reduced if the optimal battery SOC costate, once obtained, is used as a starting point in succeeding iterations.

The power-split control problem is also solved using DP. For this, the feasible SOC and ICE torque ranges are gridded with 200 and 300 points, respectively. The required optimization time of DP is much larger, 8 to 12 hours, while the solution's accuracy is lower than the accuracy of ECMS's solution, due to the discretization in both SOC and engine torque that DP requires. Therefore, in the rest of this section we show results obtained by using ECMS for the power-split control problem.

\section{B. Optimization results}

Minimization of fuel consumption is carried out in three different case studies. First, a conventional vehicle is considered and the ICE is calibrated to minimize fuel consumption on the driving cycle. Second, the conventional vehicle is converted to an HEV by mounting the EM on the rear axle. Fuel consumption is minimized by splitting the power between ICE and EM, but still using the same ICE calibration as for the conventional vehicle. Third, the ICE is recalibrated with representative operating points of the HEV. 

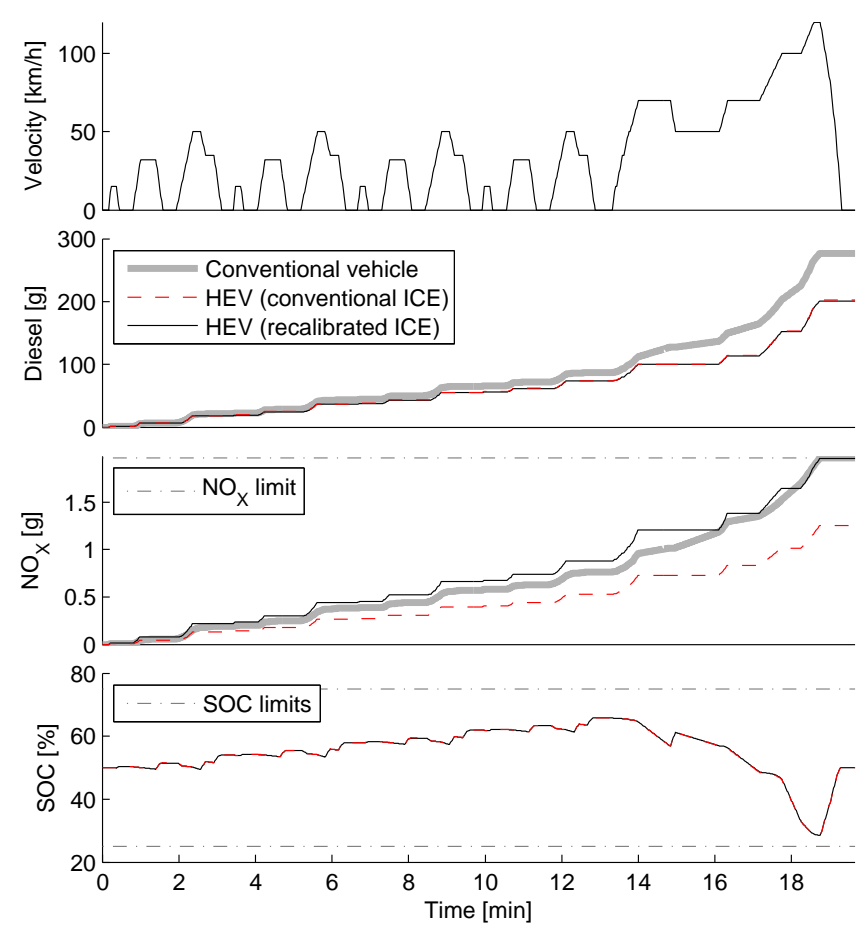

(a) New European Driving Cycle (NEDC).
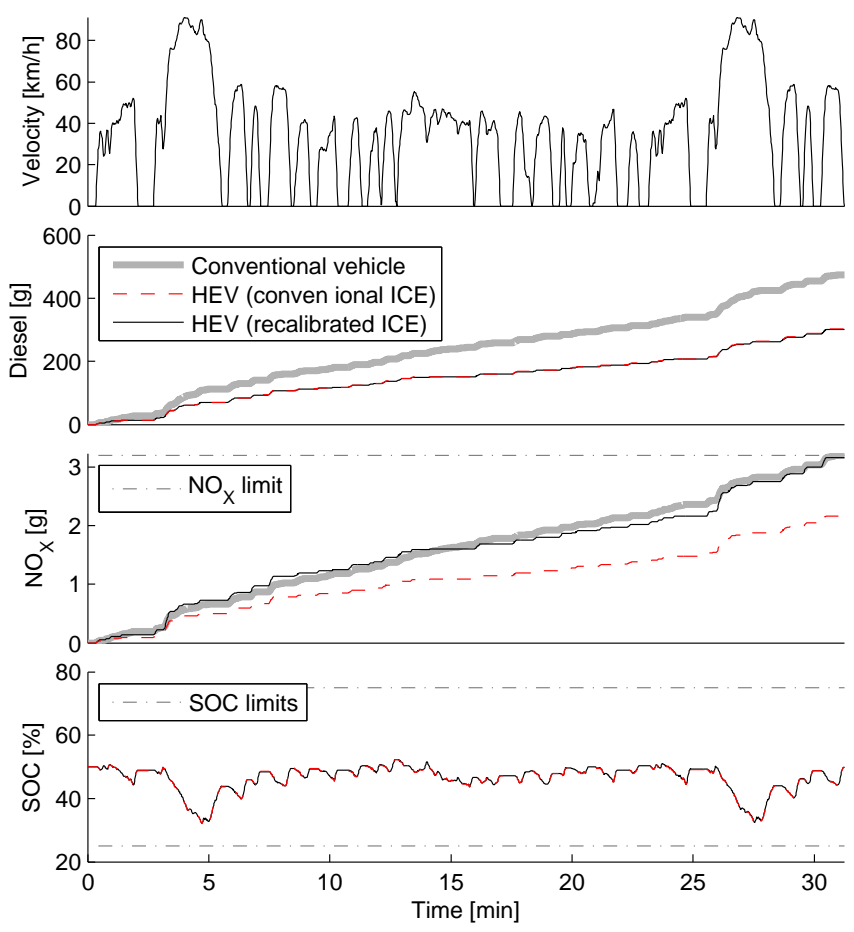

(b) USA's Federal Test Procedure (FTP75).

Fig. 9. The subplots, from top to bottom, depict demanded velocity of the driving cycle and simulation results including the optimal cumulated fuel consumption, $\mathrm{NO}_{\mathrm{X}}$ emissions, and battery SOC. The optimal fuel consumption and SOC trajectories nearly overlap, for the HEV with ICE calibrated for a conventional vehicle, and the HEV with optimally calibrated ICE.

The optimal fuel consumption and $\mathrm{NO}_{\mathrm{X}}$ emissions are shown in Table I and the optimal states' evolution is depicted in Fig. 9. It can be observed that by recalibrating the ICE using the optimal representative operating points of the HEV, fuel consumption can be decreased by $0.62 \%$ for NEDC, and $0.63 \%$ for FTP75, by still keeping the $\mathrm{NO}_{\mathrm{X}}$ emissions under the legislative limit.

The optimal ICE efficiency and $\mathrm{NO}_{\mathrm{X}}$ maps are shown in Fig. 10. The figure also depicts the optimal operating points of the conventional vehicle and the HEV. It can be observed that the HEV avoids engine operation at low torque, where the engine is less efficient. The percentage of total cycle time the engine is on in the HEV is $19.9 \%$ on the NEDC and $19 \%$ on the FTP75, for both cases of engine calibration. The average engine efficiency, when the engine delivers power on the NEDC, increased from $37.77 \%$ to $38.5 \%$, by recalibrating the ICE in the HEV. On the FTP75, the engine efficiency increased from $38.53 \%$ to $38.97 \%$.

The complete speed/torque grid used for the calibration of the fuel and $\mathrm{NO}_{\mathrm{X}}$ flow maps is illustrated in the right subplots of Fig. 10, with the shaded regions depicting the representative grid points of the driving cycles. Coincidentally, the engine operating points of the HEV, regarding the shaded regions, are distributed similarly to engine operating points of the conventional vehicle. Therefore, the recalibration only slightly enlarged the high efficiency area towards greater torque values, but besides this, the engine efficiency maps are similar.

The engine maps shown in Fig. 10 are calibrated in their entire range, although the engine operating points do not excite the entire operating range of the engine. The optimal calibration procedure makes this possible, due to the single coefficient $\lambda_{N O_{X}}^{*}$ that weights fuel consumption and emissions in the entire engine range. However, engine operating points that are not excited by the driving cycle, could be further tuned (perhaps manually), without having any impact on the net fuel consumption and emissions for that driving cycle.

The optimization problem is also solved by using the computationally efficient strategy described in Section V-A. As initial maps in the algorithm, the maps are used that are calibrated for the conventional vehicle. The algorithm converged in just two iterations, requiring only two powersplit optimizations. This outcome was also revealed in Fig. 9, where it can be observed that the last two case studies have nearly the same optimal SOC trajectories regardless of whether the ICE is calibrated for a conventional vehicle, or for the HEV. The result is obtained in less than 4 minutes and is exactly the same as the optimal result of the last case study in Table I.

\section{DISCUSSION AND FUTURE WORK}

This section discusses the process of ICE data gathering, the possibility of powertrain model enhancement and future studies.

\section{A. ICE data gathering and calibration}

The generation of the static ICE fuel and $\mathrm{NO}_{\mathrm{X}}$ flow maps may require a large number of measurements due to the many combinations of input values. To limit the number of 

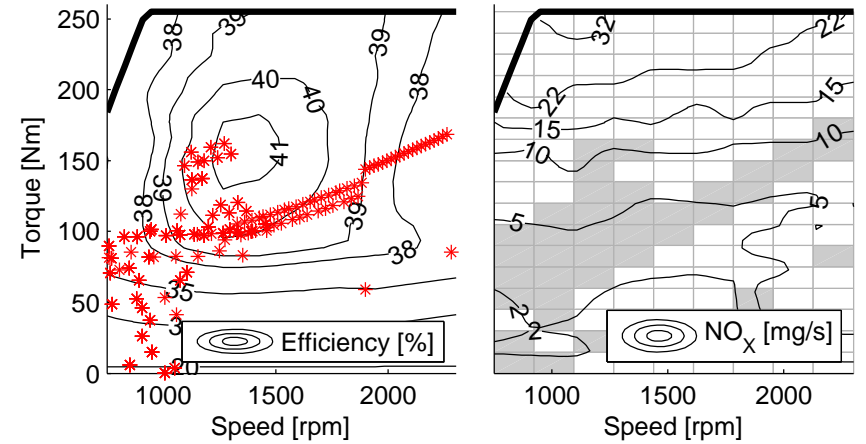

(a) ICE maps calibrated for the conventional vehicle on the NEDC.
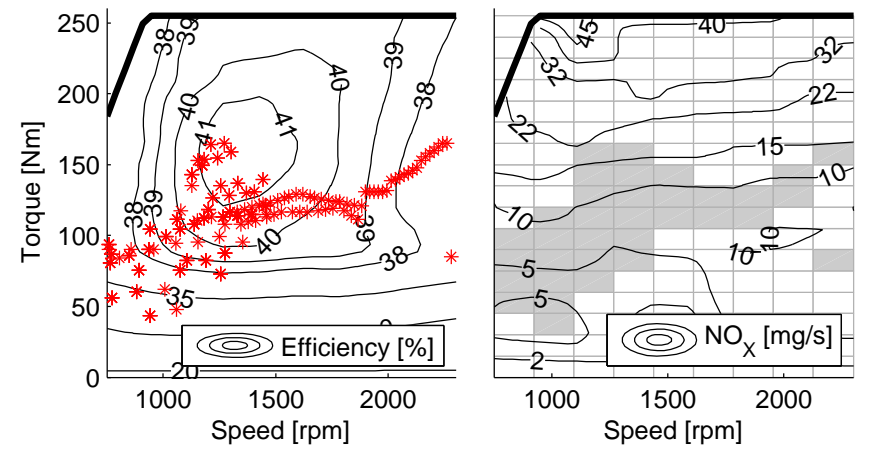

(c) ICE maps calibrated for the HEV on the NEDC.
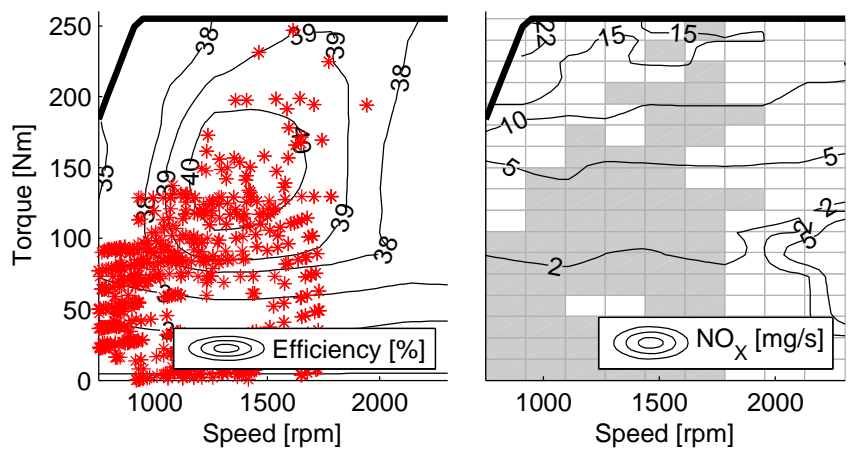

(b) ICE maps calibrated for the conventional vehicle on the FTP75.
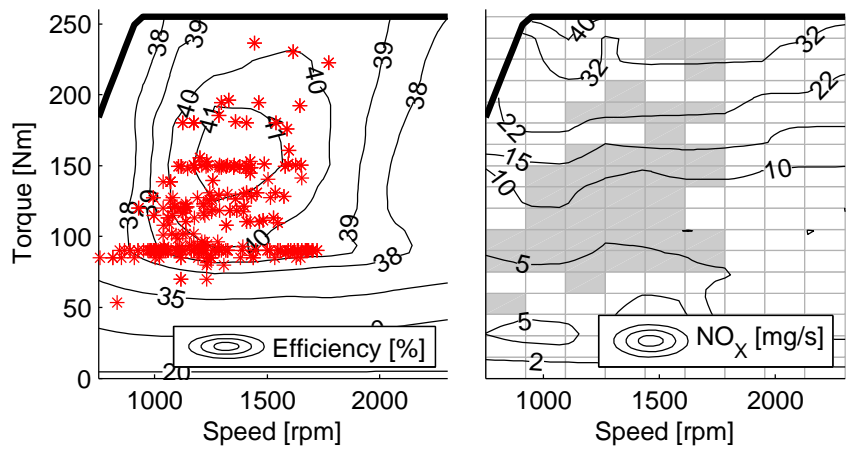

(d) ICE maps calibrated for the HEV on the FTP75.

Fig. 10. Optimal ICE efficiency map and $\mathrm{NO}_{\mathrm{X}}$ map, at the left and right subplot of each sub-figure, respectively. The optimal ICE operating points are depicted by stars. The $\mathrm{NO}_{\mathrm{X}}$ map also illustrates the complete grid of speed/torque points used for the engine calibration. The shaded regions indicate the representative grid points at which the ICE is operated for the selected driving cycle.

measurements, the ICE was operated at 2596 input combinations, found according to the D-optimal design methodology [49]. The collected data was fitted to models describing boost pressure, oxygen fraction in the intake manifold, engine torque and $\mathrm{NO}_{\mathrm{X}}$ emissions, as functions of engine speed, injected fuel and $z$ (see [50], [51], [52] for details). The maps were then enlarged to $10 \times 17 \times 30 \times 30 \times 30$ grid points using linear interpolation, and the torque map was inverted to get torque as input, rather than fuel flow.

In order to obtain more informative data for the limited amount of measurements, ICE operation at high speed and torque was not considered, but the focus was kept on the region where the studied driving cycles reside. The performed measurements covered only part of the available speed and torque range of a Volvo 2.4 liter passenger car Diesel engine (with actual maximum speed of $4700 \mathrm{rpm}$ ). This, however, does not infringe the generality of the proposed method. Utilization of the entire available speed and torque range shall be considered in future studies.

\section{B. Enhanced modeling and future studies}

In this study, we considered a relatively simple powertrain model, thus emphasizing the optimization methodology. However, the proposed method can readily be applied to more detailed powertrain models, including auxiliary losses, rotational inertia of the ICE and EM, and more detailed transmission and clutch models. Dynamic ICE start/stop, clutch and gearbox models are also possible when, for example, changes in discrete states are penalized. This will increase the computational complexity of DP when optimizing the power-split control, as additional states will be needed for the ICE on/off, clutch and transmission gear [53]. The proposed method may also employ stochastically generated driving cycles [24], applied to both HEVs and plug-in HEVs.

Constraints on other emissions, such as soot, hydrocarbon, carbon monoxide, and combustion noise, may also be considered. This will necessitate additional costates, which will increase the computational complexity [19], but the optimization method will otherwise be the same. Implementing engine thermal dynamics and transient modeling, e.g. boost pressure dynamics, is a more challenging task that shall be considered in future studies. Experimental validation of improvements from the ICE recalibration is a major topic to be also considered in future studies.

\section{CONCLUSION}

A method is presented for combined optimization of ICE management settings and power-split control of a hybrid electric vehicle. It is shown that the optimization problem is separable in space, while in the case where battery SOC limits are not activated, it is also shown that the problem is separable in time. The optimal solution is obtained by iteratively solving the power-split control problem using dynamic programming or the ECMS. A computationally efficient solution is presented, which for the studied example managed to achieve the 
TABLE II

PARAMETER VALUES.

\begin{tabular}{ll}
\hline Vehicle frontal area & $A_{f}=2.4 \mathrm{~m}^{2}$ \\
Aerodynamic drag coefficient & $c_{d}=0.36$ \\
Rolling resistance coefficient & $c_{r}=0.012$ \\
Air density & $\rho_{a}=1.184 \mathrm{~kg} / \mathrm{m}^{3}$ \\
Wheel radius & $r_{w}=0.38 \mathrm{~m}$ \\
Vehicle rotational inertia & $I_{v}=1.2 \mathrm{kgm}^{2}$ \\
\hline$u=160 \mathrm{~V}, P_{B \max }=20 \mathrm{~kW}, P_{B \min }=-36 \mathrm{~kW}, R=0.26 \Omega$, \\
$r(g=1, \ldots, 6)=\{16.8,9.6,6.3,4.7,3.5,2.7\}$ \\
$\eta(g=1, \ldots, 5)=92.15 \%, \eta(g=6)=93.1 \%$.
\end{tabular}

global optimum $25 x$ faster (i.e. $100 \mathrm{~min} / 4 \mathrm{~min}$ ) than the time needed for the full optimization, when ECMS is used for the power-split control. The speedup is $120 x$ (i.e. $8 \mathrm{~h} / 4 \mathrm{~min}$ ), when DP is used for power-split control in the full optimization.

The provided example considers optimization of engine management settings and power-split control of a parallel HEV, driven on the NEDC and FTP75. It is found that by recalibrating the engine a fuel improvement can be achieved of about $0.62 \%$, by still keeping the $\mathrm{NO}_{\mathrm{X}}$ emissions under the legislative limit. In a real vehicle the fuel improvement may differ due to dynamics that have been omitted in the simulation model. However, it is important to note that although small, the fuel improvement can be achieved with almost no additional investment cost, in contrast to the cost of electric components in the HEV.

After recalibration, all the HEV components are for sure on-board diagnosis relevant; before they may not have been since it was the calibration for the conventional vehicle. In a more general case the ICE may not be first calibrated for a conventional vehicle, but, instead, it may be calibrated directly for the HEV (consider heavily downsized ICE, or ICE designed specifically for the HEV). In this case, the method provides an automated way to perform ICE calibration for the hybrid application.

\section{APPENDIX A}

\section{DATA AND MODELING}

Given the longitudinal vehicle velocity $v$ and road gradient $\alpha$, the dissipative forces the vehicle encounters are the aerodynamic drag and the rolling resistance

$$
F_{a}=\frac{\rho_{a} A_{f} c_{d}}{2} v^{2}, \quad F_{r}=m g c_{r} \cos \alpha .
$$

Then, the demanded angular velocity and torque at the wheels are

$$
\begin{aligned}
\omega & =\frac{v}{r_{w}} \\
T & =\left(I_{v}+m r_{w}^{2}\right) \dot{\omega}+\left(F_{a}+F_{r}+m g \sin \alpha\right) r_{w} .
\end{aligned}
$$

Here, $m$ is the vehicle mass, which is $2000 \mathrm{~kg}$ for the conventional vehicle and $2340 \mathrm{~kg}$ for the HEV. The remaining parameters are given in Table II.

\section{REFERENCES}

[1] L. Guzzella and A. Sciarretta, Vehicle Propulsion Systems, 3rd ed. Berlin, Heidelberg: Springer, Verlag, 2013.

[2] A. Sciarretta and L. Guzzella, "Control of hybrid electric vehicles," IEEE Control Systems Magazine, vol. 27, no. 2, pp. 60-70, 2007.
[3] N. Murgovski, L. Johannesson, J. Sjöberg, and B. Egardt, "Component sizing of a plug-in hybrid electric powertrain via convex optimization," Journal of Mechatronics, vol. 22, no. 1, pp. 106-120, 2012.

[4] M. Pourabdollah, N. Murgovski, A. Grauers, and B. Egardt, "Optimal sizing of a parallel PHEV powertrain," IEEE Transactions on Vehicular Technology, vol. 62, no. 6, pp. 2469-2480, 2013.

[5] B. Egardt, N. Murgovski, M. Pourabdollah, and L. Johannesson, "Electromobility studies based on convex optimization: Design and control issues regarding vehicle electrification," IEEE Control Systems Magazine, vol. 34, no. 2, pp. 32-49, 2014.

[6] N. J. Schouten, M. A. Salman, and N. A. Kheir, "Energy management strategies for parallel hybrid vehicles using fuzzy logic," Control Engineering Practice, vol. 11, no. 2, pp. 171-177, 2003.

[7] P. Pisu and G. Rizzoni, "A comparative study of supervisory control strategies for hybrid electric vehicles," IEEE Transactions on Control Systems Technology, vol. 15, no. 3, pp. 506-518, 2007.

[8] L. Wu, Y. Wang, X. Yuan, and Z. Chen, "Multiobjective optimization of HEV fuel economy and emissions using the self-adaptive differential evolution algorithm," IEEE Transactions on Vehicular Technology, vol. 60, no. 6, pp. 2458-2470, 2011.

[9] M. Sorrentino, G. Rizzo, and I. Arsie, "Analysis of a rule-based control strategy for on-board energy management of series hybrid vehicles," Control Engineering Practice, vol. 19, no. 12, pp. 1433-1441, 2011.

[10] J. S. Martnez, R. I. John, D. Hissel, and M.-C. Péra, "A survey-based type-2 fuzzy logic system for energy management in hybrid electrical vehicles," Information Sciences, vol. 190, no. 0, pp. 192-207, 2012.

[11] L. Johannesson, M. Åsbogård, and B. Egardt, "Assessing the potential of predictive control for hybrid vehicle powertrains using stochastic Dynamic Programming," IEEE Transactions on Intelligent Transportation Systems, vol. 8, no. 1, pp. 71-83, 2007.

[12] L. Johannesson, S. Pettersson, and B. Egardt, "Predictive energy management of a 4QT series-parallel hybrid electric bus," Control Engineering Practice, vol. 17, no. 12, pp. 1440-1453, 2009.

[13] N. Murgovski, J. Sjöberg, and J. Fredriksson, "A methodology and a tool for evaluating hybrid electric powertrain configurations," Int. J. Electric and Hybrid Vehicles, vol. 3, no. 3, pp. 219-245, 2011.

[14] T. V. Johnson, "Vehicular emissions in review," SAE Int. J. Engines, vol. 5, no. 2, pp. 216-234, 2013.

[15] J. B. Heywood, Internal Combustion Engine Fundamentals. McGrawHill, 1988.

[16] C. Lin, H. Peng, J. W. Grizzle, and J. Kang, "Power management strategy for a parallel hybrid electric truck," IEEE Transactions on Control Systems Technology, vol. 11, no. 6, pp. 839-849, 2003.

[17] M. Koot, J. T. B. A. Kessels, B. de Jager, W. P. M. H. Heemels, P. P. J. van den Bosch, and M. Steinbuch, "Energy management strategies for vehicular electric power systems," IEEE Transactions on Vehicular Technology, vol. 54, no. 3, pp. 771-782, 2005.

[18] E. Rishavy, S. Hamilton, J. Ayers, and M. Keane, "Engine control optimization for best fuel economy with emission constraints," SAE International, Technical Paper 770075, 1977.

[19] H. S. Rao, A. I. Cohen, J. A. Tennant, and K. L. V. Voorhies, "Engine control optimization via nonlinear programming," SAE International, Technical Paper 790177, 1979.

[20] G. Schmitz, U. Oligschläger, and G. Eifles, "Automated system for optimized calibration of engine management systems," SAE International, Technical Paper 940151, 1994.

[21] H.-M. Koegler, A. Fuerhapter, M. Mayer, and K. Gschweitl, "DGIengine calibration, using new methodology with CAMEO," SAE International, Technical Paper 2001-24-0012, 2001.

[22] H. Altenstrasser, Y. Kato, N. Keuth, and T. Winsel, "MiL-based calibration and validation of Diesel-ECU models using emission and fuel consumption prediction during dynamic warm-up tests (NEDC)," SAE International, Technical Paper 2012-01-0432, 2012.

[23] I. Kolmanovsky, M. van Nieuwstadt, and J. Sun, "Optimization of complex powertrain systems for fuel economy and emissions," in Proceedings of the 1999 IEEE International Conference on Control Applications, Kohala Coast-Island of Hawai, USA, 1999.

[24] I. Kolmanovsky, I. Siverguina, and B. Lygoe, "Optimization of powertrain operating policy for feasibility assessment and calibration: Stochastic Dynamic Programming approach," in Proceedings of the American Control Conference, Anchorage, AK, 2002.

[25] J. Kang, I. Kolmanovsky, and J. Grizzle, "Dynamic optimization of lean burn engine aftertreatment," Journal of Dynamics Systems, Measurement and Control, vol. 123, no. 2, pp. 153-160, 2001.

[26] A. E. Bryson and Y.-C. Ho, Applied Optimal Control. Taylor \& Francis Group, 1975. 
[27] L. S. Pontryagin, V. G. Boltyanskii, R. V. Gamkrelidze, and E. F. Mishchenko, The Mathematical Theory of Optimal Processes, L. W. Neustadt, Ed. Interscience Publishers, 1962.

[28] R. Bellman, Dynamic Programming. New Jersey: Princeton Univ Pr, 1957.

[29] L. Guzzella and C. H. Onder, Introduction to Modeling and Control of Internal Combustion Engine Systems. Springer-Verlag, 2010.

[30] M. Grahn, "Model-based Diesel engine management system optimization. A strategy for transient engine operation," Ph.D. dissertation, Chalmers University of Technology, Gothenburg, Sweden, 2013.

[31] ICCT, "World-harmonized light-duty vehicles test procedure (WLTP)," International Council on Clean Transportation, Tech. Rep., 2013.

[32] I. Brahma and J. Chi, "Development of a model-based transient calibration process for Diesel engine electronic control module tables - Part 1: data requirements, processing, and analysis," International Journal of Engine Research, vol. 13, no. 1, pp. 77-96, 2012.

[33] - "Development of a model-based transient calibration process for Diesel engine electronic control module tables - Part 2: modelling and optimization," International Journal of Engine Research, vol. 13, no. 2, pp. 147-168, 2012.

[34] M. Grahn, K. Johansson, and T. McKelvey, "A Diesel engine management system strategy for transient engine operation," in 7th IFAC Symposium on Advances in Automotive Control, Tokyo, Japan, 2013.

[35] $\_$, "Model-based diesel engine management system optimization for transient engine operation," Control Engineering Practice, vol. 29, pp. 103-114, 2014.

[36] D. Alberer and L. Re, "Optimization of the transient Diesel engine operation,” SAE International, Technical Paper 2009-24-0113, 2009.

[37] - "Fast oxygen based transient Diesel engine operation," SAE Int. J. Engines, vol. 2, no. 1, pp. 405-413, 2009.

[38] M. Benz, M. Hehn, C. H. Onder, and L. Guzzella, "Model-based actuator trajectories optimization for a Diesel engine using a direct method," $J$. Eng. Gas Turbines Power, vol. 133, no. 3, 2010.

[39] S. Nakayama, T. Fukuma, A. Matsunaga, T. Miyake, and T. Wakimoto, "A new dynamic combustion control method based on charge oxygen concentration for Diesel engines," SAE International, Technical Paper 2003-01-3181, 2003.

[40] S. Nakayama, T. Ibuki, H. Hosaki, and H. Tominaga, "An application of model based combustion control to transient cycle-by-cycle Diesel combustion," SAE Int. J. Engines, vol. 1, no. 1, pp. 850-860, 2008.

[41] S. Chen and O. Yanakiev, "Transient NOx emission reduction using exhaust oxygen concentration based control for a Diesel engine," SAE International, Technical Paper 2005-01-0372, 2005.

[42] C. Atkinson and G. Mott, "Dynamic model-based calibration optimization: An introduction and application to Diesel engines," SAE International, Technical Paper 2005-01-0026, 2005.

[43] C. Atkinson, M. Allain, and H. Zhang, "Using model-based rapid transient calibration to reduce fuel consumption and emissions in Diesel engines," SAE International, Technical Paper 2008-01-1365, 2008.

[44] G. Paganelli, S. Delprat, T. M. Guerra, J. Rimaux, and J. Santin, "Equivalent consumption minimization strategy for parallel hybrid powertrains," in IEEE Vehicular Technology Conference, 2002, pp. 2076-2081.

[45] S. Delprat, J. Lauber, T. M. Guerra, and J. Rimaux, "Control of a parallel hybrid powertrain: optimal control," IEEE Transactions on Vehicular Technology, vol. 53, no. 3, pp. 872-881, 2004.

[46] A. Sciarretta, L. Guzzella, and M. Back, "A real-time optimal control strategy for parallel hybrid vehicles with on-board estimation of control parameters," in Proc. IFAC Symp. Advances Automotive Control, Salerno, Italy, 2004.

[47] C. Musardo, G. Rizzoni, and B. Staccia, "A-ECMS: An adaptive algorithm for hybrid electric vehicle energy management," in 44th IEEE Conference on Decision and Control, and the European Control Conference, Seville, Spain, 2005.

[48] P. Rutquist, C. Breitholtz, and T. Wik, "On the infinite time solution to state-constrained stochastic optimal control problems," Automatica, vol. 44, no. 7, pp. 1800-1805, 2008.

[49] D. C. Montgomery, Design and Analysis of Experiments, 7th ed. John Wiley \& Sons, Inc., 2009.

[50] M. Grahn, K. Johansson, C. Vartia, and T. McKelvey, "A structure and calibration method for data-driven modeling of NOx and soot emissions from a Diesel engine," SAE International, Technical Paper 2012-010355, 2012.

[51] M. Grahn, K. Johansson, and T. McKelvey, "B-splines for Diesel engine emission modeling," in IFAC Workshop on Engine and Powertrain Control, Simulation and Modeling (ECOSM), Rueil-Malmaison, France, 2012.

[52] — "Data-driven emission model structures for diesel engine management system development," International Journal of Engine Research, 2014.

[53] D. F. Opila, X. Wang, R. McGee, R. B. Gillespie, J. A. Cook, and J. W. Grizzle, "An energy management controller to optimally trade off fuel economy and drivability for hybrid vehicles," IEEE Transactions on Control Systems Technology, vol. 20, no. 6, pp. 1490-1505, 2012. 